\title{
An unusual cutaneous manifestation of ulcerative colitis: thrombotic skin gangrene
}

\author{
Suveer Singh, Kai Noshirwani
}

\begin{abstract}
Summary
Ulcerative colitis has a number of associated skin lesions. We report the case of a patient with active ulcerative disease presenting with rapidly progressive necrotic skin lesions, identified as thrombotic and requiring skin grafting. Thrombotic cutaneous gangrene is a rare but potentially serious manifestation of uncontrolled ulcerative colitis.
\end{abstract}

Keywords: ulcerative colitis, microvascular thrombosis, skin gangrene

A 57-year-old Caucasian man with a 10-year history of controlled ulcerative colitis presented with right iliac fossa pain and bloody diarrhoea. Colonoscopy revealed inflammation extending throughout the large bowel and biopsies were consistent with ulcerative colitis. He was commenced on intravenous steroids.

Over the previous 10 years, his ulcerative colitis had been well controlled on aminosalicyclic acids. However, during this period he had been admitted several times with venous occlusions, three deep vein thromboses in the legs, a right axillary vein occlusion and more recently a suspected right gluteal vein thrombosis. His long-term anticoagulation had been stopped seven years earlier for unspecified reasons.

Three days following admission, he developed a painful superficial haemorrhagic area below the left nipple. Within 24 hours it had progressed to occupy the left anterior aspect of the abdominal wall (figure). The next day two similar lesions appeared in the right loin and nipple areas, respectively. A swelling of the right leg was confirmed as a deep vein thrombosis on venography. He was anticoagulated with intravenous heparin as a 5000-unit loading dose followed by an infusion of 1400 units $/ h$, adjusted to maintain an activated partial thromboplastin time at 2-2.5 times the base value. ${ }^{11}$

Investigations revealed a normocytic anaemia
A putative diagnosis of skin infarction secondary to thrombosis of small cutaneous vessels was made. It was hoped that intravenous heparin for the deep vein thrombosis would also help his skin lesions. While anticoagulated, the cutaneous lesions did not progress, and in fact the smaller lesions appeared to regress two days into treatment. However, the initial skin lesion broke down, requiring a full thickness skin graft. It is likely that this area had already infarcted prior to the commencement of heparin.

Despite the presence of active colitis, blood loss during anticoagulation was not a major problem, as evidenced by stable haemoglobin levels. However, his ulcerative colitis did not settle with medical treatment, and he underwent a total colectomy six weeks after admission.

\section{Discussion}

Cutaneous manifestations of ulcerative colitis include erythema nodosum, erythema multiforme or the potentially serious pyoderma gangrenosum, which is the most specific of its

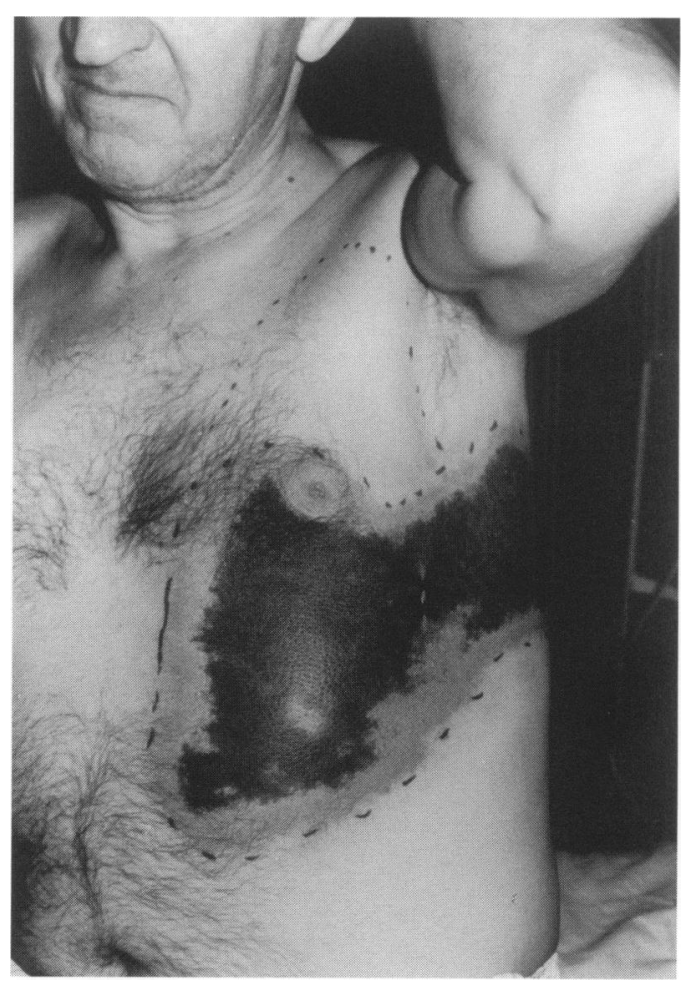

Figure Superficial haemorrhagic infarct over left anterior chest wall \begin{abstract}
(haemoglobin $11.3 \mathrm{~g} / \mathrm{dl}$ ), white cell count $13 \times 10^{9} \mathrm{~g} / 1$ and normal platelet count, erythrocyte sedimentation rate, coagulation screen, cryoglobulins, anticardiolipin antibody titres, proteins $\mathrm{C}$ and $\mathrm{S}$ and antithrombin III levels, prior to anticoagulation. Skin biopsy of the haemorrhagic lesion demonstrated a full thickness infarction, capilliritis, and microthrombi in the dermal vessels with extensive erythrocyte extravasation; the findings were nonspecific but not consistent with pyoderma gangrenosum.

Medicine, Kingston Kingston upon S Singh

Correspondence to $\mathrm{D}$ Suveer Singh, Guy's Hospital, St Thomas Street, Accepted 12 January 1996
\end{abstract}




\section{Summary points \\ - there is an increased thrombophilic tendency in ulcerative colitis \\ - thrombotic skin gangrene is a rare cutaneous manifestation of ulcerative colitis, histologically distinct from pyoderma gangrenosum \\ - progression to full thickness skin infarction is a potentially serious complication \\ - a thrombophilia screen and skin biopsy should be performed prior to early anticoagulation}

skin associations. Thrombotic cutaneous gangrene is a rare complication of ulcerative colitis, quite distinct histologically from pyoderma gangrenosum, and has been reported infrequently to date. Similar lesions have been described in the past ${ }^{1,2}$ and more recently by Stapleton et al, ${ }^{3}$ who treated their patient's skin condition successfully with intravenous heparin prior to the development of full thickness infarction.

Microvascular focal thrombosis is thought to be the basis of this cutaneous gangrene. ${ }^{4}$ There is an increased incidence of thromboembolic phenomena associated with ulcerative colitis. Five per cent of ulcerative colitis sufferers develop deep vein thrombosis, whilst there are reports of increased cerebral venous thromboses. ${ }^{5}$ This increased clotting tendency may result from raised levels of clotting factors $V$,

1 Bargen JA, Barker NW. Extensive arterial and venous thrombosis complicating chronic ulcerative colitis. Arch Intern Med 1936; 58: 17-31.

2 Spitell JA, Owen CA, Thompson JH, Sauer WG. Hypercoagulability and thrombosis in chronic ulcerative colitis Collected papers in medicine, Mayo Clin 1964; 53: Vol. 53-7.

3 Stapleton SR, Curley RK, Simpson WA. Cutaneous gangrene secondary to focal thrombosis. Clin Exp Dermatol 1989 14: $87-9$.

4 Ball GV, Goldman LN. Chronic ulcerative colitis, skin necrosis and cryofibrinogenaemia. Ann Intern Med 1976; 85 464-6.

5 Musio F, Older SA, Jenkins T, Gregorie EM. Case report: cerebral venous thrombosis as a manifestation of acute ulcerative colitis. Am f Sci 1993; 305: 28-35.

6 Schneiderman JH, Sharp JA, Sutton DMC. Cerebral and retinal vascular complications of inflammatory bowel disease. Ann Neurol 1979; 5: 331 - 7 .
VIII and fibrinogen ${ }^{6,8}$ and a fall in antithrombin III levels due to protein-losing enteropathy, ${ }^{7}$ while circulating cryoglobulins are another potential cause of cutaneous gangrene. Furthermore, recent reports have shown increased platelet counts in ulcerative colitisassociated thromboembolic disease. ${ }^{9}$

The management of such gangrenous skin lesions is not clearly established, although reports of resolution following prednisolone and intravenous heparin ${ }^{10}$ have set a basis for treatment. Bleeding complications due to anticoagulation in the presence of active colitis appear not to have arisen from previous reports. However, should significant bleeding be a problem, there is some evidence of a reduced haemorrhagic tendency and similar $\vec{O}$ efficacy of low molecular weight heparin as an alternative to unfractionated heparin, albeit in other settings.

We reiterate the importance of early suspicion of cutaneous gangrene if unusual skin lesions, that do not conform to pyoderma ? gangrenosum, appear in inflammatory bowel of disease. A full thrombophilic blood screen should be done, and early anticoagulation with $N$ heparin, aiming to prevent progressive cuta- 0 neous infarction. The role of intravenous steroids in the treatment of ulcerative colitisassociated cutaneous gangrene remains unclear, other than for treating the exacerbation of colitis.

7 Knot E, Ten Cate JW, Leeksma O, Tytgar GN, Vreeken No evidence for a prethrombotic state in stable chronic inflammatory bowel disease. 7 Clin Pathol 1983; 36: 1387 90.

8 Lam A, Borda IT, Inwood MJ, Thomson S. Coagulation studies in ulcerative colitis and Crohn's disease. Gastroenterology 1975; 68: 245-51.

9 Webberley MJ, Hart MT, Melikian V. Thromboembolism in inflammatery bowel disease: the role of platelets. Gut in inflammatory bow; 34: 247-51.

10 Wackers FJ, Tytgar GN, Vreeken J. Necrotizing vasculitis and ulcerative colitis. $B M \mathcal{F}$ 1974; 4: 83-4

11 Fennerty A, Campbell IA, Routledge PA. Anticoagulants in venous thromboembolism. $B M F$ 1988; 297: 1285-8. 